\title{
Correction to: Effectiveness of a new model of primary care management on knee pain and function in patients with knee osteoarthritis: Protocol for THE PARTNER STUDY
} (1) cossuate

\author{
D. J. Hunter ${ }^{1,2^{*}}$, R. S. Hinman³, J. L. Bowden', T. Egerton ${ }^{3}$, A. M. Briggs ${ }^{4}$, S. J. Bunker ${ }^{5}$, J. Kasza ${ }^{6}$, A. B. Forbes ${ }^{6}$, \\ S. D. French ${ }^{7,8}$, M. Pirotta ${ }^{9}$, D. J. Schofield ${ }^{10}$, N. A. Zwarr ${ }^{11,12}$, K. L. Bennell ${ }^{13}$ and the PARTNER Study Team
}

\section{Erratum: BMC Musculoskeletal Disorders https://doi.org/10.1186/s12891-018-2048-0}

After the publication of this protocol [1], our collaborator Prima Health solutions advised us of their intent to withdraw from the study. Their primary role was to provide remotely delivered weight-loss services (via the Healthy Weight for Life program) to eligible participants in the intervention group. These services were partly provided as in-kind and partly funded through the study. We have received ethical approval from the University of Sydney to replace the Healthy Weight for Life program with the Commonwealth Scientific and Industrial Research Organisation's (CSIRO) Total Wellbeing Diet. The amended weight loss advice and support paragraph of the manuscript is outlined below. All changes to the protocol were made and approved before starting the trial and were prospectively changed on our trial registration (ACT RN12617001595303).

Amended weight loss advice and support paragraph: If the patient has a BMI $\geq 27 \mathrm{~kg} / \mathrm{m}^{2}$, the patient will be offered the option of participating in the remotelydelivered weight loss program. The Australian Commonwealth Scientific and Industrial Research Organisation's (CSIRO) "Total Wellbeing Diet" is based on an evidence-based weight management strategy that utilises a structured, nutritionally balanced eating plan designed to be incorporated into a balanced

\footnotetext{
* Correspondence: david.hunter@sydney.edu.au

${ }^{1}$ Institute of Bone and Joint Research, Kolling Institute, University of Sydney, Sydney, Australia

2Department of Rheumatology, Royal North Shore Hospital, Sydney, NSW, Australia
}

lifestyle program $[2,3]$. The program is a 12 - week, low glycaemic index, high protein, healthy eating program with online support and tracking tools, meal plans and educational resources on a healthy diet. It is delivered by SP Health (http://www.sphealth.com/) on behalf of the CSIRO. After completion of the 12-week program, patients may elect to continue the basic program for an additional 12-weeks. Patients who elect to undertake the online weight-loss program will continue to be supported by the PARTNER Care Support Team throughout their time on the weight-loss program. This program will be undertaken in conjunction with the PARTNER exercise program and educational resources on healthy lifestyle change.

\begin{abstract}
Author details
${ }^{1}$ Institute of Bone and Joint Research, Kolling Institute, University of Sydney, Sydney, Australia. ${ }^{2}$ Department of Rheumatology, Royal North Shore Hospital, Sydney, NSW, Australia. ${ }^{3}$ Centre for Health, Exercise and Sports Medicine, Department of Physiotherapy, The University of Melbourne, Melbourne, Victoria, Australia. ${ }^{4}$ School of Physiotherapy and Exercise Science, Curtin University, Perth, WA, Australia. ${ }^{5}$ Department of Physiotherapy, The University of Melbourne, Melbourne, Victoria, Australia. 'Biostatistics Unit, School of Public Health and Preventive Medicine, Monash University, Melbourne, Victoria, Australia. ${ }^{\top}$ School of Rehabilitation Therapy, Queen's University, Kingston, Ontario, Canada. ${ }^{8}$ Department of Chiropractic, Faculty of Science and Engineering, Macquarie University, Sydney, NSW, Australia. 'Department of General Practice, The University of Melbourne, Melbourne, Victoria, Australia. ${ }^{10}$ Department of Economics, Faculty of Business and Economics, Macquarie University, Sydney, Australia. ${ }^{11}$ School of Public Health and Community Medicine, University of New South Wales, Sydney, NSW, Australia. ${ }^{12}$ School of Medicine, University of Wollongong, Wollongong, NSW, Australia. ${ }^{13}$ Centre for Health, Exercise and Sports Medicine, Department of Physiotherapy, The University of Melbourne, Melbourne, Victoria, Australia.
\end{abstract}

Published online: 20 December 2018

\section{References}

1. Hunter DJ, et al. Effectiveness of a new model of primary care management on knee pain and function in patients with knee osteoarthritis: Protocol for 
THE PARTNER STUDY. BMC Musculoskelet Disord. 2018;19:132 https://doi. org/10.1186/s12891-018-2048-0.

2. Noakes M, Clifton PM. The CSIRO Total Wellbeing Diet. Australia: Penguin Books; 2005.

3. Noakes M, Keogh JB, Foster PR, Clifton PM. Effect of an energy-restricted, high-protein, low-fat diet relative to a conventional high-carbohydrate, lowfat diet on weight loss, body composition, nutritional status, and markers of cardiovascular health in obese women. Am J Clin Nutr. 2005;81 (6):1298-306. 